\title{
ENHANCED BINDING REVISITED FOR A SPINLESS PARTICLE IN NON-RELATIVISTIC QED
}

\author{
ISABELLE CATTO, PAVEL EXNER AND CHRISTIAN HAINZL
}

\begin{abstract}
We consider a spinless particle coupled to a quantized Bose field and show that such a system has a ground state for two classes of short-range potentials which are alone too weak to have a zero-energy resonance.
\end{abstract}

\section{INTRODUCTION AND MAIN RESULTS}

Let us begin by specifying the classes of potentials to be considered. Throughout the paper we suppose that (i) $V \in L^{\infty}$ is nonzero and attractive, $V \leq 0$, and (ii) $V$ belongs to $L^{3 / 2}\left(\mathbb{R}^{3}\right)$, which is well known to ensure, in particular, that $V$ lies in the Rollnik class, i.e.

$$
\|V\|_{R}^{2}:=\iint_{\mathbb{R}^{6}} \frac{|V(x)||V(y)|}{|x-y|^{2}} d x d y<+\infty .
$$

Finally, we adopt one of the following assumptions: (iii) $V$ is strictly attractive, $V<0$, and satisfies the inequality

$$
|\Delta V| \leq C|V|,
$$

with a positive constant $C$, or alternatively (iii') $V$ is compactly supported with $\Delta V$ integrable. We will say more on these assumptions in the remarks following Theorem 1 below.

We denote by $H_{\lambda}$ the family of Schrödinger operators $H_{\lambda}:=p^{2}+\lambda V$ on $L^{2}\left(\mathbb{R}^{3}\right)$ for positive parameters $\lambda$. Its eigenvalues are monotonically decreasing functions of $\lambda$ in $[0,+\infty)$, and it is well-known that at some positive critical value $\lambda_{0}$, which is called the "coupling-constant threshold" an eigenvalue emerges from the continuum. More precisely, if $\lambda \leq \lambda_{0}, H_{\lambda}$ has no negative eigenvalues, whereas when $\lambda>\lambda_{0}$ it has at least one bound state.

We now couple this Schrödinger operator to the radiation field and consider the so-called Pauli-Fierz operator

$$
H_{\alpha}^{V}=(p+\sqrt{\alpha} A(x))^{2}+H_{\mathrm{f}}+\lambda V,
$$

Date: November 6, 2018.

Key words and phrases. QED, Self-energy, Enhanced binding, Coupling constants.

C.H. acknowledges support through the European Union's IHP network Analysis \& Quantum HPRN-CT-2002-00277. 
acting on the Hilbert space

$$
\mathcal{H}=L^{2}\left(\mathbb{R}^{3} ; \mathbb{C}\right) \otimes \mathcal{F}
$$

where $\mathcal{F}=\bigoplus_{n=0}^{+\infty} L_{b}^{2}\left(\mathbb{R}^{3 n} ; \mathbb{C}\right)$ is the Fock space for the photon field and $L_{b}^{2}\left(\mathbb{R}^{3 n}\right)$ is the space of symmetric functions in $L^{2}\left(\mathbb{R}^{3 n}\right)$ representing $n$ photon states, with $n=0$ corresponding to the one-dimensional vacuum sector. Following the usual convention we abuse notation and use the same symbol for $H_{\mathrm{f}}$ and $\overline{I \otimes H_{\mathrm{f}}}$, etc. The operator $H_{\alpha}^{V}$ is essentially self-adjoint on $\mathcal{D}(\Delta) \cap \mathcal{D}\left(H_{\mathrm{f}}\right)$, where the symbol $\mathcal{D}$ denotes the operator domain - see [BFS].

We denote the ground state energy as

$$
E(\alpha, \lambda V):=\inf \operatorname{spec} H_{\alpha}^{V},
$$

and the spectrum is then the half-line $[E(\alpha, \lambda V),+\infty)$, see again BFS. In GLL, Theorem 3.1], Griesemer, Lieb and Loss show under rather weak assumptions about the potential $V$ that, in the case when $\lambda>\lambda_{0}$, i.e. when the Schödinger operator $-\Delta+\lambda V$ has a ground state, it persists after coupling to the radiation field. Moreover, in [HVV], Hainzl, Vougalter and Vugalter prove for a particular class of potentials that in the case when $\lambda=\lambda_{0}$, the coupling to the field can create a ground state for small coupling constant $\alpha$, despite the fact that the underlying Schödinger operator $-\Delta+$ $\lambda_{0} V$ has no ground state. Recall also that in the case of a particle with spin the same result was proved by two of the present authors in $\mathrm{CH}$; a different proof is given in [CVV].

Our goal here is to show that $E(\alpha, \lambda V)$ is an eigenvalue of $H_{\alpha}^{V}$ for coupling in some interval $\left(\gamma, \lambda_{0}\right]$, with $\lambda_{0}-\gamma=\mathcal{O}(\alpha)$ for $\alpha$ small. The strategy of proof will be based on the expansion of the self-energy in powers of $\alpha$, as provided in [H1, H2, HVV, $\mathrm{CH}$, and by checking that the Griesemer-LiebLoss criterium (i.e., inequality (2.1) below) is satisfied for $\alpha$ small enough. To this aim we use a result of Klaus and Simon [KS], which allows us to demonstrate the enhanced binding for the class of potentials indicated above; we need not assume like in [HVV] that the Hamiltonian has a zero-energy resonance in the absence of the field.

Recall that in the dipole approximation, in the case of a large coupling $\alpha$ the enhanced binding was shown earlier by Hiroshima and Spohn HiSp, see also $\mathrm{AA}]$ in the context of linear coupling.

We fix units in such a way that the Planck constant $\hbar=1$, the speed of light $c=1$, and the electron mass $m=\frac{1}{2}$. The electron charge is then given by $e=\sqrt{\alpha}$, with $\alpha \approx 1 / 137$ being the fine structure constant. In the present paper $\alpha$ plays the role of a small, dimensionless number which measures the coupling to the radiation field. Our results hold for sufficiently small values of $\alpha$. The operator $p=-\mathrm{i} \nabla$ is the electron momentum while 
$A$ is the quantized magnetic vector potential, which is given by

$$
A(x)=\sum_{\lambda=1,2} \int_{\mathbb{R}^{3}} \frac{\chi(|k|)}{2 \pi|k|^{1 / 2}} \varepsilon^{\lambda}(k)\left[a_{\lambda}(k) \mathrm{e}^{\mathrm{i} k \cdot x}+a_{\lambda}^{*}(k) \mathrm{e}^{-\mathrm{i} k \cdot x}\right] \mathrm{d} k,
$$

where the annihilation and creation operators $a_{\lambda}$ and $a_{\lambda}^{*}$, respectively, satisfy the usual commutation relations

$$
\left[a_{\nu}(k), a_{\lambda}^{*}(q)\right]=\delta(k-q) \delta_{\lambda, \nu},
$$

and

$$
\left[a_{\lambda}(k), a_{\nu}(q)\right]=0, \quad\left[a_{\lambda}^{*}(k), a_{\nu}^{*}(q)\right]=0 .
$$

In the following we use the notation

$$
A(x)=D(x)+D^{*}(x) .
$$

The vectors $\varepsilon^{\lambda}(k) \in \mathbb{R}^{3}$ in $A(x)$ are orthonormal polarization vectors perpendicular to $k$ which are chosen in a such a way that

$$
\varepsilon^{2}(k)=\frac{k}{|k|} \wedge \varepsilon^{1}(k) .
$$

The function $\chi(|k|)$ describes the ultraviolet cutoff for the interaction at large wave-numbers $k$. For the sake of simplicity we choose for $\chi$ the Heaviside function $\Theta(\Lambda-|k|)$; more general cut-off functions would work, however, let us emphasize the fact that we shall sometimes use the radial symmetry of $\chi$ in the proofs. Throughout the paper we assume $\Lambda=1$. This corresponds to the energy $m c^{2}$ in our system of units and represents a natural upper bound to which the validity of non-relativistic QED can be extended.

The photon field energy $H_{\mathrm{f}}$ is given by

$$
H_{\mathrm{f}}=\sum_{\lambda=1,2} \int_{\mathbb{R}^{3}}|k| a_{\lambda}^{*}(k) a_{\lambda}(k) \mathrm{d} k
$$

and the field momentum reads

$$
P_{\mathrm{f}}=\sum_{\lambda=1,2} \int_{\mathbb{R}^{3}} k a_{\lambda}^{*}(k) a_{\lambda}(k) \mathrm{d} k .
$$

Since $\mathcal{H}$ can be also written as $\bigoplus_{n=0}^{+\infty} L^{2}\left(\mathbb{R}^{3} ; \mathbb{C}\right) \otimes L_{b}^{2}\left(\mathbb{R}^{3 n} ; \mathbb{C}\right)$ we can express a general vector $\Psi \in \mathcal{H}$ as a direct sum

$$
\Psi=\bigoplus_{n=0}^{\infty} \psi_{n}
$$

where $\psi_{n}=\psi_{n}\left(x, k_{1}, \ldots, k_{n}\right)$ is an $n$-photon state. For simplicity, we do not include the variables corresponding to the polarization of the photons.

To simplify further the notation, we observe that the Hamiltonian is translation invariant and introduce the unitary transformation

$$
U=\mathrm{e}^{\mathrm{i} P_{\mathrm{f}} \cdot x}
$$

acting on $\mathcal{H}$. Since

$$
U A(x) U^{*}=A(0)
$$


and

we obtain

$$
U p U^{*}=p-P_{\mathrm{f}}
$$

$$
U H_{\alpha}^{V} U^{*}=\left(p-P_{\mathrm{f}}+\sqrt{\alpha} A\right)^{2}+H_{\mathrm{f}}+\lambda V,
$$

where $A=A(0)$. The operator $U$ preserves spectral properties, in particular,

$$
\inf \operatorname{spec}\left[U H_{\alpha}^{V} U^{*}\right]=\inf \operatorname{spec} H_{\alpha}^{V} \text {. }
$$

Thus we shall rather work with $U H_{\alpha}^{V} U^{*}$ in the following; abusing the notation we will use again the symbol $H_{\alpha}^{V}$ for it.

Our main result is the following

THEOREM 1. Adopt the assumptions (i), (ii), and either (iii) or (iii'). Then there exists a function $g: \mathbb{R}^{+} \rightarrow(0,1)$ such that for any small enough $\alpha$ and all $\lambda \in\left(\lambda_{0}(1-g(\alpha)), \lambda_{0}\right]$, the spectral threshold $E(\alpha, \lambda V)$ is an eigenvalue of $H_{\alpha}^{V}$.

REMARK 1. The assumptions combine different type of requirements. For instance, (1.1) or $\Delta V \in L^{1}$ impose restrictions mainly on local regularity of the potential. On the other hand, (ii) regulates its decay; on a heuristic level one may say that the potential should behave as $|x|^{-2-\epsilon}$ at infinity.

REMARK 2. It comes out of the proof that $g(\alpha)$ is of order of $\alpha$, more specifically, the relation (2.37) shows that $g(\alpha)=c \alpha+\mathcal{O}\left(\alpha^{2} \ln \alpha\right)$ with $c>0$ holds as $\alpha \rightarrow 0$. It is important that we get in this way an asymptotical lower bound to $g(\alpha)$ which allows us to assess how much the binding is enhanced.

REMARK 3. In connection with the previous remark we want to emphasize that all the constants appearing in the proof can be evaluated explicitly. Assuming that we choose a potential $V$ such that the constants $C, b(V)$ in (1.1) and (2.39), respectively, are of order of one, it turns out that Theorem 1 holds for $\alpha \lesssim 10^{-2}$ which covers the physically important case.

REMARK 4. We have recalled above the results [GLL] and [HVV], the latter using the existence of a zero-energy resonance state together with a continuity argument which shows that $H_{\alpha}^{V}$ has a bound state for values of $\lambda$ slightly below $\lambda_{0}$. Our strategy is similar but the proof is more constructive, in particular, it provides a rough estimate on how far below $\lambda_{0}$ one can descend to still ensure the existence of a ground state. In addition our method covers a different and in several respects wider class of potentials $V$, in particular, we require neither a compact support nor the radial symmetry of the potential.

REMARK 5. Using the methods of $[\mathrm{CH}]$, Theorem 1 can also be proven for the case of particles with spin. Unfortunately the numbers of inequalities needed increase dramatically. For that reason we restricted our attention to the more convenient case of bosons. 


\section{PROOF OF THEOREM 1}

Let $0<\lambda \leq \lambda_{0}$. According to Griesemer, Lieb and Loss GLL the ground state exists provided

$$
E(\alpha, \lambda V)<E(\alpha, 0),
$$

where $E(\alpha, 0)$ is the electron self-energy. Thus we are going to construct a trial state $\Psi \in L^{2}\left(\mathbb{R}^{3}\right) \otimes \mathcal{F}$ which ensures that the last inequality is satisfied,

$$
\left(\Psi ; H_{\alpha}^{V} \Psi\right)<E(\alpha, 0)\|\Psi\|^{2} .
$$

The strategy of proof is as follows: we will compare the respective expansions of $E(\alpha, 0)$ and $\left(\Psi ; H_{\alpha}^{V} \Psi\right)$ for the trial state $\Psi$ in terms of the coupling constant $\alpha$. From [H1, HVV] we already know the first three terms in the Taylor expansion of the former, namely

$$
\left|E(\alpha, 0)-\alpha \pi^{-1}+\alpha^{2}\left\langle 0\left|D D \mathcal{A}_{\alpha}^{-1} D^{*} D^{*}\right| 0\right\rangle\right| \leq C_{\text {self }} \alpha^{3},
$$

for some positive constant $C_{\text {self }}$, where $|0\rangle$ is the vacuum vector, $\langle\cdot ; \cdot\rangle$ denotes the scalar product in the photon Fock space $\mathcal{F}$, and with

$$
\mathcal{A}_{\alpha}=P_{\mathrm{f}}^{2}+H_{\mathrm{f}}+2 \alpha D^{*} D \text {. }
$$

Recall that actually in $\mathrm{HVV}$ the proof is given for $\mathcal{A}_{0}$ instead of $\mathcal{A}_{\alpha}$ in the second-order term in (2.3), but the same argument carries through mutatis mutandis to the present case.

Consider now a quantity $g(\alpha) \in(0,1)$, to be determined later, and observe that when the coupling parameter $\lambda$ satisfies

$$
(1-g(\alpha)) \lambda_{0}<\lambda \leq \lambda_{0}
$$

then the Schrödinger operator

$$
h_{\alpha}^{\lambda}:=-(1-g(\alpha)) p^{2}+\lambda V
$$

has a negative eigenvalue $e_{\lambda}(\alpha):=-\left|e_{\lambda}\right|$ at the bottom of the spectrum. This trivially follows from the inequality $\frac{\lambda}{1-g(\alpha)}>\lambda_{0}$ and our choice of $\lambda_{0}$ to be critical. We denote by $\psi_{\lambda}$ a corresponding eigenstate which may be chosen without loss of generality as real-valued and normalized in $L^{2}$. Our trial function, to be inserted into (2.2), will involve only two photons being of the form

$$
\Psi=\psi_{\lambda} \oplus \psi_{1} \oplus \psi_{2}
$$

with

$$
\psi_{1}=-2 \sqrt{\alpha} L^{-1} D^{*} p \psi_{\lambda}
$$

and

$$
\psi_{2}=-\alpha L^{-1} D^{*} D^{*} \psi_{\lambda} .
$$

The operator $L$ on $L^{2}\left(\mathbb{R}^{3}\right) \otimes \mathcal{F}$ appearing in here is defined by

$$
L=(1-g(\alpha))\left(p-P_{\mathrm{f}}\right)^{2}+\lambda V+\left|e_{\lambda}\right|+H_{\mathrm{f}}+2 \alpha D^{*} D ;
$$

the definitions (2.5b) and (2.5c) make sense because $L$ is invertible on the orthogonal complement of the vacuum sector $L^{2}\left(\mathbb{R}^{3}\right) \otimes \mathbb{C}|0\rangle$; this follows from the fact that it is unitarily equivalent to $h_{\alpha}^{\lambda}+\left|e_{\lambda}\right|+H_{\mathrm{f}}+2 \alpha D^{*}(x) D(x)$ by 
means of the operator (1.9). Note that, with the abuse of notation mentioned in the opening, we often use $\psi_{\lambda}$ as a shorthand for $\psi_{\lambda} \otimes|0\rangle$.

The canonical commutation relations yield the identity

$$
A^{2}=D^{*} D^{*}+D D+2 D^{*} D+\frac{1}{\pi} ;
$$

using it together with commutativity of $P_{\mathrm{f}}$ with $D, D^{*}$, we find that

$$
\begin{aligned}
& \left(\Psi ; H_{\alpha}^{V} \Psi\right)=g(\alpha)\|P \Psi\|^{2}+\left[\frac{\alpha}{\pi}-\left|e_{\lambda}\right|\right]\|\Psi\|^{2} \\
& +(\Psi ; L \Psi)+2 \Re\left(\Psi ;\left[2 \sqrt{\alpha} P D^{*}+\alpha D^{*} D^{*}\right] \Psi\right)+\left(\psi_{\lambda} ;\left(h_{\alpha}^{\lambda}+\left|e_{\lambda}\right|\right) \psi_{\lambda}\right),
\end{aligned}
$$

where $P:=p-P_{\mathrm{f}}$ denotes the total momentum; the last term in the righthand side of (2.7) cancels by definition of $e_{\lambda}, h_{\alpha}^{\lambda}$ and $\psi_{\lambda}$.

Let us further remark that the argument will require - similarly as in the previous works [HS, $\mathrm{CH}, \mathrm{HHS}$ - to replace the field Hamiltonian $H_{\mathrm{f}}$ by $\tilde{H}_{\mathrm{f}}:=H_{\mathrm{f}}+\alpha^{3}$ in order to deal with the logarithmically divergent infrared terms; this would amount to adding an extra $-\alpha^{3}\|\Psi\|^{2}$ term at the righthand side of (2.7). Observe that by our choice of $\Psi$ the identity

$$
\begin{aligned}
&(\Psi ; L \Psi)+ 2 \Re\left(\Psi ;\left[2 \sqrt{\alpha} P D^{*}+\alpha D^{*} D^{*}\right] \Psi\right)=\left(\psi_{\lambda} ; L \psi_{\lambda}\right) \\
&-2 \Re\left(\psi_{\lambda} ;-\alpha D D L^{-1}(-2 \sqrt{\alpha} P D)^{*} L^{-1}(-2 \sqrt{\alpha} P D)^{*} \psi_{\lambda}\right) \\
&-\left\|L^{-1 / 2}\left[2 \sqrt{\alpha} P D^{*}+\alpha D^{*} D^{*}\right] \psi_{\lambda}\right\|^{2}
\end{aligned}
$$

holds, and the same is true if we replace $L$ in the last relation by $\tilde{L}$ referring to $\tilde{H}_{\mathrm{f}}$. Thus we obtain

$$
\begin{gathered}
\left(\Psi ; \tilde{H}_{\alpha}^{V} \Psi\right)=g(\alpha)\|P \Psi\|^{2}+\left[\frac{\alpha}{\pi}-\left|e_{\lambda}\right|-\alpha^{3}\right]\|\Psi\|^{2}-4 \alpha\left\|\tilde{L}^{-1 / 2} D^{*} p \psi_{\lambda}\right\|^{2} \\
-\alpha^{2}\left(\psi_{\lambda} ; D D \tilde{L}^{-1} D^{*} D^{*} \psi_{\lambda}\right)+8 \alpha^{2} \Re\left(\tilde{L}^{-1} D^{*} D^{*} \psi_{\lambda} ; P D^{*} \tilde{L}^{-1} D^{*} p \psi_{\lambda}\right)
\end{gathered}
$$

where $\tilde{H}_{\alpha}^{V}$ refers again to $\tilde{H}_{\mathrm{f}}$. On the other hand, apart of taming the infrared singularity the extra term is irrelevant, as long as we looking for an effect of order of $\alpha^{2}$. This is why we will abuse notation writing non-tilded quantities everywhere except the one place below where this Hamiltonian shift indeed matters.

To estimate the last term at the right-hand side of (2.9), notice that the Cauchy-Schwarz inequality yields

$$
\begin{aligned}
& 8 \alpha^{2}\left|\left(L^{-1} D^{*} D^{*} \psi_{\lambda} ; P D^{*} L^{-1} D^{*} p \psi_{\lambda}\right)\right| \\
& \quad \leq \frac{4 \alpha^{3}}{a}\left\|L^{-1 / 2} P D L^{-1} D^{*} D^{*} \psi_{\lambda}\right\|^{2}+4 \alpha a\left\|L^{-1 / 2} D^{*} p \psi_{\lambda}\right\|^{2}
\end{aligned}
$$


with a positive constant $a$ to be chosen later. The last term can be combined with the similar term in (2.9) giving

$$
\begin{gathered}
\left(\Psi ; H_{\alpha}^{V} \Psi\right) \leq g(\alpha)\|P \Psi\|^{2}-\left|e_{\lambda}\right|\|\Psi\|^{2}+\frac{\alpha}{\pi}\|\Psi\|^{2}-4 \alpha(1-a)\left\|L^{-1 / 2} D^{*} p \psi_{\lambda}\right\|^{2} \\
\quad-\alpha^{2}\left(\psi_{\lambda} ; D D L^{-1} D^{*} D^{*} \psi_{\lambda}\right)+\frac{4 \alpha^{3}}{a}\left\|L^{-1 / 2} P D L^{-1} D^{*} D^{*} \psi_{\lambda}\right\|^{2} .
\end{gathered}
$$

To estimate further the terms appearing in (2.10) we need a series of technical lemmata.

LEMMA 2.1. The following inequality holds,

$$
\left(\psi_{\lambda} ; D D L^{-1} D^{*} D^{*} \psi_{\lambda}\right) \geq\left\langle 0\left|D D \mathcal{A}_{\alpha}^{-1} D^{*} D^{*}\right| 0\right\rangle+g(\alpha) C_{1},
$$

where

$$
C_{1}:=\| P_{\mathrm{f}} \mathcal{A}_{\alpha}^{-1} D^{*} D^{*}|0\rangle \|^{2}
$$

Proof. We denote $L=\mathcal{Q}+b$, with

$$
\mathcal{Q}=(1-g(\alpha))\left(p^{2}+P_{\mathrm{f}}^{2}\right)+\lambda V+\left|e_{\lambda}\right|+H_{\mathrm{f}}+2 \alpha D^{*} D
$$

and $b=-2(1-g(\alpha)) p P_{f}$, and we use twice the second resolvent equation, $(\mathcal{Q}+b)^{-1}=\mathcal{Q}^{-1}-\mathcal{Q}^{-1} b \mathcal{Q}^{-1}+\mathcal{Q}^{-1} b(\mathcal{Q}+b)^{-1} b \mathcal{Q}^{-1} \geq \mathcal{Q}^{-1}-\mathcal{Q}^{-1} b \mathcal{Q}^{-1}$

where the inverse of $\mathcal{Q}+b$ and the last inequality makes sense in the complement to the vacuum sector where the operator is strictly positive as we have remarked above. Hence we have

$$
\begin{aligned}
\left(\psi_{\lambda} ; D D L^{-1} D^{*} D^{*} \psi_{\lambda}\right) & \geq\left(\psi_{\lambda} ; D D \mathcal{Q}^{-1} D^{*} D^{*} \psi_{\lambda}\right) \\
+ & 2(1-g(\alpha))\left(\psi_{\lambda} ; D D \mathcal{Q}^{-1} p P_{\mathrm{f}} \mathcal{Q}^{-1} D^{*} D^{*} \psi_{\lambda}\right) .
\end{aligned}
$$

Furthermore, the second term at the right-hand side vanishes. To check this claim, recall that $\psi_{\lambda}$ belongs by construction to the null-space of $h_{\alpha}^{\lambda}+\left|e_{\lambda}\right|$, and that $h_{\alpha}^{\lambda}$ commutes with the operator

$$
\mathcal{K}:=(1-g(\alpha)) P_{\mathrm{f}}^{2}+H_{\mathrm{f}}+2 \alpha D^{*} D .
$$

It follows easily that

$$
\mathcal{Q}^{-1} D^{*} D^{*} \psi_{\lambda}=\mathcal{K}^{-1} D^{*} D^{*} \psi_{\lambda}
$$

and therefore

$$
\left(\psi_{\lambda} ; D D \mathcal{Q}^{-1} p P_{\mathrm{f}} \mathcal{Q}^{-1} D^{*} D^{*} \psi_{\lambda}\right)=\left(\psi_{\lambda} ; p \psi_{\lambda}\right)\left\langle 0\left|D D \mathcal{K}^{-1} P_{\mathrm{f}} \mathcal{K}^{-1} D^{*} D^{*}\right| 0\right\rangle=0,
$$

because $\psi_{\lambda}$ is real-valued as indicated above making the first factor zero.

Using (2.15) again, we find that the first term reads

$$
\begin{gathered}
\left(\psi_{\lambda} ; D D \mathcal{Q}^{-1} D^{*} D^{*} \psi_{\lambda}\right)=\left(\psi_{\lambda} ; D D \mathcal{K}^{-1} D^{*} D^{*} \psi_{\lambda}\right) \\
=\left\|\psi_{\lambda}\right\|^{2}\left\langle 0\left|D D \mathcal{K}^{-1} D^{*} D^{*}\right| 0\right\rangle \\
\geq\left\langle 0\left|D D \mathcal{A}_{\alpha}^{-1} D^{*} D^{*}\right| 0\right\rangle+g(\alpha)\left\langle 0\left|D D \mathcal{A}_{\alpha}^{-1} P_{\mathrm{f}}^{2} \mathcal{A}_{\alpha}^{-1} D^{*} D^{*}\right| 0\right\rangle
\end{gathered}
$$


where in the last line we used the fact that $\psi_{\lambda}$ is normalized together with the second resolvent equation and positivity of $\mathcal{K}$ in the complement of the vacuum sector. Hence the claim.

LEMMA 2.2. For any positive constant $\mu$ we have

$$
\begin{aligned}
& \left\|L^{-1 / 2} D^{*} p \psi_{\lambda}\right\|^{2} \\
& \quad \geq\left[C_{4}(\mu)+\mu C_{3}(\mu)\right]\left\|p \psi_{\lambda}\right\|^{2}-\frac{\lambda}{2} C_{3}(\mu)\left(\psi_{\lambda} ; \Delta V \psi_{\lambda}\right),
\end{aligned}
$$

with

and

$$
C_{3}(\mu)=\frac{2}{3}\left\langle 0\left|D(\mathcal{K}+\mu)^{-2} D^{*}\right| 0\right\rangle
$$

$$
C_{4}(\mu)=\frac{2}{3}\left\langle 0\left|D(\mathcal{K}+\mu)^{-1} D^{*}\right| 0\right\rangle .
$$

Proof. Using the relation analogous to (2.13), we find

$$
\begin{aligned}
& p D L^{-1} D^{*} p \geq p D(\mathcal{K}+\mu)^{-1} D^{*} p \\
& \quad-p D(\mathcal{K}+\mu)^{-1}\left[-2 p P_{\mathrm{f}}(1-g(\alpha))+h_{\alpha}^{\lambda}+\left|e_{\lambda}\right|-\mu\right](\mathcal{K}+\mu)^{-1} D^{*} p .
\end{aligned}
$$

Again, since $\psi_{\lambda}$ is real valued the term containing $p P_{\mathrm{f}}$ vanishes; notice that the same conclusion can also be made using symmetry of the cut-off function. Since $\mathcal{K}$ acts on photon variables whereas $h_{\alpha}^{\lambda}$ acts on those of the electron, the two operators commute and the second term at the right-hand side of the last estimate can be rewritten as

$$
\begin{array}{r}
\left(\psi_{\lambda} ; p D(\mathcal{K}+\mu)^{-1}\left[-2 p P_{\mathrm{f}}(1-g(\alpha))+h_{\alpha}^{\lambda}+\left|e_{\lambda}\right|-\mu\right](\mathcal{K}+\mu)^{-1} D^{*} p \psi_{\lambda}\right) \\
=C_{3}(\mu)\left(p \psi_{\lambda} ;\left(h_{\alpha}^{\lambda}+\left|e_{\lambda}\right|-\mu\right) p \psi_{\lambda}\right)
\end{array}
$$

with $C_{3}(\mu)$ given by (2.18). Observing that $\psi_{\lambda}$ belongs to the null-space of $h_{\alpha}^{\lambda}+\left|e_{\lambda}\right|$ we can further cast a part of the last expression into the form

$$
\left(p \psi_{\lambda} ;\left(h_{\alpha}^{\lambda}+\left|e_{\lambda}\right|\right) p \psi_{\lambda}\right)=-\frac{1}{2}\left(\psi_{\lambda} ;\left[p,\left[p, h_{\alpha}^{\lambda}+\left|e_{\lambda}\right|\right]\right] \psi_{\lambda}\right)=\frac{\lambda}{2}\left(\psi_{\lambda} ; \Delta V \psi_{\lambda}\right)
$$

to obtain

$$
\left(p \psi_{\lambda} ;\left(h_{\alpha}^{\lambda}+\left|e_{\lambda}\right|-\mu\right) p \psi_{\lambda}\right)=-\mu\left\|p \psi_{\lambda}\right\|^{2}+\frac{\lambda}{2}\left(\psi_{\lambda} ; \Delta V \psi_{\lambda}\right) .
$$

On the other hand,

$$
\left(\psi_{\lambda} ; p D(\mathcal{K}+\mu)^{-1} D^{*} p \psi_{\lambda}\right)=C_{4}(\mu)\left\|p \psi_{\lambda}\right\|^{2}
$$

with $C_{4}(\mu)$ given by (2.19), which concludes the proof.

Our next auxiliary result is the following:

LEMMA 2.3. Let $\alpha>0$ and $0<\lambda \leq \lambda_{0}$, then for any positive number $g(\alpha)<1-\frac{\lambda}{\lambda_{0}}$ there are positive constants $\beta$ and $C(V)$ such that

$$
p^{2} \leq \beta\left(h_{\alpha}^{\lambda}+\left|e_{\lambda}\right|\right)+C(V) .
$$


Proof. For the inequality (2.22) to hold, the constant $\beta$ which appears at the right-hand side has obviously to satisfy the inequality $\beta>\frac{1}{1-g(\alpha)}$, or equivalently $\beta(1-g(\alpha))-1>0$. We fix an arbitrary $\beta$ with this property, to be specified later. Next we notice that inequality (2.22) will follow from

$$
p^{2}+\lambda \tilde{V} \geq-\tilde{C}(V)
$$

with

$$
\tilde{V}:=\frac{1}{1-g(\alpha)-\beta^{-1}} V, \quad \tilde{C}(V):=\frac{C(V)}{\beta(1-g(\alpha))-1},
$$

because the last inequality is equivalent to (2.22) with the term $\left|e_{\lambda}\right|$ at the right-hand side neglected. In other words, it is sufficient that the Schrödinger operator $p^{2}+\lambda \tilde{V}$ has no spectrum below $-\tilde{C}(V)$. From the proof of the Birman-Schwinger bound in [RS, Theorem XIII.10] and the fact that $\tilde{V}$ is non-positive it follows that this happens if and only if

$$
\frac{\lambda^{2}}{16 \pi^{2}} \iint_{\mathbb{R}^{6}} \frac{|\tilde{V}(x)||\tilde{V}(y)|}{|x-y|^{2}} \mathrm{e}^{-2 \sqrt{\tilde{C}(V)}|x-y|} d x d y<1 .
$$

Let us denote by $\mathcal{K}_{m}$ the function $x \mapsto \frac{\mathrm{e}^{-\sqrt{m}}|x|}{4 \pi|x|^{2}}$ with a fixed positive $m$ which represents the resolvent kernel in the above expression; it is clear that $\mathcal{K}_{m}$ belongs to $L^{1}\left(\mathbb{R}^{3}\right)$ and $\int_{\mathbb{R}^{3}} \mathcal{K}_{m}(x) d x=\frac{1}{\sqrt{m}}$. We employ these observations in the following chain of inequalities,

$$
\begin{aligned}
& \frac{\lambda^{2}}{16 \pi^{2}} \iint_{\mathbb{R}^{6}} \frac{|\tilde{V}(x)||\tilde{V}(y)|}{|x-y|^{2}} \mathrm{e}^{-2 \sqrt{\tilde{C}(V)}|x-y|^{2}} d x d y \\
& \quad=\frac{\lambda^{2}}{4 \pi\left(1-g(\alpha)-\beta^{-1}\right)^{2}} \int_{\mathbb{R}^{3}}\left(V \star K_{4 \tilde{C}(V)}\right)(x) V(x) d x \\
& \leq \frac{\lambda^{2}}{4 \pi\left(1-g(\alpha)-\beta^{-1}\right)^{2}}\|V\|_{L^{2}}^{2}\left\|K_{4} \tilde{C}(V)\right\|_{L^{1}} \\
& \leq \frac{\lambda^{2}}{8 \pi\left(1-g(\alpha)-\beta^{-1}\right)^{2} \sqrt{\tilde{C}(V)}}\|V\|_{L^{2}}^{2},
\end{aligned}
$$

where in the second and third line we used Cauchy-Schwarz and Young inequalities, respectively. Thus the bound (2.23) will be satisfied if the last expression is smaller than one. For a fixed $\beta>2(1-g(\alpha))^{-1}$ we can estimate $\left(1-g(\alpha)-\beta^{-1}\right)^{-1 / 2}$ by $\sqrt{\beta}$, and consequently, the inequality (2.22) will hold uniformly in $\lambda \in\left[0 ; \lambda_{0}\right)$, as long as the positive constant $C(V)$ is chosen large enough to satisfy

$$
\frac{\lambda_{0}^{2} \sqrt{\beta}\|V\|_{L^{2}}^{2}}{8 \pi\left(1-g(\alpha)-\beta^{-1}\right)^{3 / 2}}<\sqrt{\tilde{C}(V)},
$$

what we set out to prove. 
Note that the constants can be chosen explicitly. The left-hand side of (2.24) diverges as $\beta \rightarrow \infty$ and in the allowed interval it has a unique minimum at $\beta=\frac{4}{1-g(\alpha)}$ where it attains the value $\frac{2 \lambda_{0}^{2}\|V\|_{L^{2}}^{2}}{3 \sqrt{3} \pi(1-g(\alpha))^{2}}$. In other words, the lemma is valid for this $\beta$ and

$$
C(V)=\left(\frac{2 \lambda_{0}^{2}\|V\|_{L^{2}}^{2}}{3 \pi(1-g(\alpha))^{2}}\right)^{2} .
$$

The reader may wonder that we have not used here fully our assumptions about the potential because for a bounded function $V \in L^{2}$ is a weaker requirement than $V \in L^{3 / 2}$, however, without the latter our main premise about existence of the coupling constant threshold may not be valid.

LEMMA 2.4. The following estimates hold:

$$
\left\|P L^{-1} D^{*} D^{*} \psi_{\lambda}\right\|^{2} \leq C_{5}
$$

and

$$
\left\|P L^{-1} D^{*} p \psi_{\lambda}\right\|^{2} \leq C_{6}(\alpha)\left\|p \psi_{\lambda}\right\|^{2}
$$

with positive $C_{5}$ and $C_{6}(\alpha)$ given in (2.28) and (2.29) below, depending on $\beta$ and $C(V)$ of the previous lemma. Using the shifted Hamiltonian $\tilde{H}_{\mathrm{f}}:=$ $H_{\mathrm{f}}+\alpha^{3}$, we have $C_{6}(\alpha) \sim \ln \left(\alpha^{-3}\right)$ as $\alpha \rightarrow 0+$.

Proof. By means of (2.22) we get the estimates

$$
L^{-1} P^{2} L^{-1} \leq L^{-1}\left[\beta\left(h_{\alpha}^{\lambda}+\left|e_{\lambda}\right|\right)+C(V)\right] L^{-1} \leq \frac{\beta}{2} H_{\mathrm{f}}^{-1}+C(V) H_{\mathrm{f}}^{-2},
$$

valid in the appropriate part of the state space, namely when sandwiched between vectors annulated by $P_{\mathrm{f}}$; in the second inequality we used the fact that for any pair of commuting operators $B, C$ with $C$ strictly positive we have $(B+C)^{-1} B(B+C)^{-1} \leq \frac{1}{2} C^{-1}$. In this way we arrive at

$$
\begin{aligned}
\left\|P L^{-1} D^{*} D^{*} \psi_{\lambda}\right\|^{2} & \leq\left[\frac{\beta}{2}\left\langle 0\left|D D H_{\mathrm{f}}^{-1} D^{*} D^{*}\right| 0\right\rangle+C(V)\left\langle 0\left|D D H_{\mathrm{f}}^{-2} D^{*} D^{*}\right| 0\right\rangle\right] \\
& :=C_{5}
\end{aligned}
$$

and

$$
\begin{aligned}
\left\|P L^{-1} D^{*} p \psi_{\lambda}\right\|^{2} & \leq\left\|p \psi_{\lambda}\right\|^{2} \frac{2}{3}\left[\frac{\beta}{2}\left\langle 0\left|D H_{\mathrm{f}}^{-1} D^{*}\right| 0\right\rangle+C(V)\left\langle 0\left|D H_{\mathrm{f}}^{-2} D^{*}\right| 0\right\rangle\right] \\
& :=\left\|p \psi_{\lambda}\right\|^{2} C_{6}(\alpha) .
\end{aligned}
$$

Now we come to the place where the shift matters because without it the right-hand side of (2.29) is infrared divergent. With the replacement $H_{\mathrm{f}} \rightarrow$ 
$H_{\mathrm{f}}+\alpha^{3}$ we have

$$
\begin{aligned}
&\langle 0| D {\left[H_{\mathrm{f}}+\alpha^{3}\right]^{-2} D^{*}|0\rangle } \\
& \quad=8 \pi \int_{0}^{1} d|k| \frac{|k|}{\left[|k|+\alpha^{3}\right]^{2}}=8 \pi\left(\ln \left(\alpha^{-3}\right)+\alpha^{3}-1\right),
\end{aligned}
$$

which concludes the argument.

Finally we come to our last technical result.

LEMMA 2.5. Under the assumption (iii') there is a positive constant $C$ such that

$$
\left|\left(\psi_{\lambda} ; \Delta V \psi_{\lambda}\right)\right| \leq C\left(\psi_{\lambda} ;|V| \psi_{\lambda}\right) .
$$

Proof. Since $V \in L^{\infty}$ the ground state is represented on $G:=\operatorname{supp} V$ by a positive smooth function. Hence $\rho(\lambda):=\sup _{G} \psi_{\lambda}\left(\inf _{G} \psi_{\lambda}\right)^{-1}$ makes sense and satisfies $1 \leq \rho(\lambda)<\infty$; the same is true for $\lambda=(1-g(\alpha)) \lambda_{0}$ corresponding to the zero-energy resonance. Using the standard estimate RS, Thm. IX.28] one can check that $\lambda \mapsto \psi_{\lambda}$ is continuous in the $\|\cdot\|_{\infty}$ norm which implies continuity of the function $\rho$. Consequently, there are positive $m$ and $M$ such that

$$
0<m \leq \tilde{\psi}_{\lambda}(x) \leq M<\infty
$$

holds for all $\left.x \in G, \lambda \in] \lambda_{0}(1-g(\alpha)) ; \lambda_{0}\right]$, and a suitable family of nonnormalized solutions (for $\psi_{\lambda}$ both the infimum and supremum vanish, of course, as we approach the zero-energy resonance). It follows that

$$
\left(\tilde{\psi}_{\lambda} ; \Delta V \tilde{\psi}_{\lambda}\right) \leq M^{2}\|\Delta V\|_{L^{1}}
$$

while $\left(\tilde{\psi}_{\lambda} ;|V| \tilde{\psi}_{\lambda}\right) \geq m^{2}\|V\|_{L^{1}}$ is positive, so it can majorize (2.33) when multiplied by a sufficiently large $C$.

Now we are ready to complete the proof of the theorem. From the definitions of $\psi_{1}$ and $\psi_{2}$ and with the help of (2.25) and (2.26) we get

$$
g(\alpha)\|P \Psi\|^{2} \leq g(\alpha)\left(1+4 \alpha C_{6}(\alpha)\right)\left\|p \psi_{\lambda}\right\|^{2}+\alpha^{2} g(\alpha) C_{5} .
$$

Using our assumptions about $V$ we can write

$$
\left(\psi_{\lambda} ;|V| \psi_{\lambda}\right)=-\left(\psi_{\lambda} ; V \psi_{\lambda}\right)=(1-g(\alpha))\left\|p \psi_{\lambda}\right\|^{2}+\left|e_{\lambda}\right|,
$$

which yields an estimate to the last term at the right-hand side of (2.17),

$$
\begin{aligned}
& \left\|L^{-1 / 2} D^{*} p \psi_{\lambda}\right\|^{2} \\
& \quad \geq\left[C_{4}(\mu)+\mu C_{3}(\mu)-\frac{\lambda}{2} C_{3}(\mu) C(1-g(\alpha))\right]\left\|p \psi_{\lambda}\right\|^{2}-\frac{\lambda}{2} C_{3}(\mu) C\left|e_{\lambda}\right| ;
\end{aligned}
$$

in case of (iii) this follows from (1.1), whereas for (iii') we employ Lemma 2.5 Next we insert into (2.12) from (2.34) and (2.35); in combination with 
Lemma 2.1 we obtain

$$
\begin{aligned}
& \left(\Psi ; H_{\alpha}^{V} \Psi\right) \\
& \leq \quad \frac{\alpha}{\pi}\|\Psi\|^{2}-\alpha^{2}\left\langle 0\left|D D \mathcal{A}_{\alpha}^{-1} D^{*} D^{*}\right| 0\right\rangle \\
& \quad-\left|e_{\lambda}\right|\|\Psi\|^{2}+\alpha\left|e_{\lambda}\right| 2 \lambda C_{3}(\mu) C(1-a) \\
& \quad+\left[g(\alpha)\left(1+4 \alpha C_{6}(\alpha)-2 \alpha(1-a) \lambda C_{3}(\mu) C\right)\right. \\
& \left.\quad \quad-4 \alpha(1-a)\left[C_{4}(\mu)+\mu C_{3}(\mu)-\frac{\lambda}{2} C_{3}(\mu) C\right]\right]\left\|p \psi_{\lambda}\right\|^{2} \\
& \quad+\alpha^{2} g(\alpha) C_{5}-\alpha^{2} g(\alpha) C_{1}+ \\
& \quad+\frac{4 \alpha^{3}}{a}\left\|L^{-1 / 2} P D L^{-1} D^{*} D^{*} \psi_{\lambda}\right\|^{2}
\end{aligned}
$$

Notice first that the term (2.36e) behaves as $\mathcal{O}\left(\alpha^{3}\right)$ for $\alpha \rightarrow 0$ which follows, e.g., from HHS Lemma 15 (v)]; thus it is irrelevant for the argument in the same way as the shift coming from the infrared regularization.

The main idea is now to choose the function $g(\alpha)$ in such a way that it cancels the factor in front of $\left\|p \psi_{\lambda}\right\|^{2}$ in (2.36c); this yields

$$
g(\alpha)=\frac{4 \alpha(1-a)\left[C_{4}(\mu)+\mu C_{3}(\mu)-\frac{\lambda}{2} C_{3}(\mu) C\right]}{1+4 \alpha C_{6}(\alpha)-2 \alpha(1-a) \lambda C_{3}(\mu) C} .
$$

We choose also $\mu=\frac{\lambda}{2} C$ and fix the parameter in (2.10) by setting

$$
1-a:=\min \left\{\left(4 C_{4}(\mu)\right)^{-1}, C_{6}(\alpha)\left(\mu C_{3}(\mu)\right)^{-1}, 3 / 4\right\} ;
$$

this yields $g(\alpha) \leq \alpha$ which means that $(2.36 \mathrm{~d})=\mathcal{O}\left(\alpha^{3}\right)$.

On the other hand, since

$$
\begin{aligned}
\|\Psi\|^{2} & =1+4 \alpha\left\|L^{-1} D^{*} p \psi_{\lambda}\right\|^{2}+\alpha^{2}\left\|L^{-1} D^{*} D^{*} \psi_{\lambda}\right\|^{2} \\
& =1+\mathcal{O}(\alpha),
\end{aligned}
$$

we deduce from (2.3) that

$$
\text { (2.36a }=E(\alpha, 0)\|\Psi\|^{2}+\mathcal{O}\left(\alpha^{3}\right) .
$$

We denote by $E(\beta)$ the bottom of the spectrum of $p^{2}+\beta V$, i.e.

$$
E(\beta):=\inf \operatorname{spec}\left(p^{2}+\beta V\right) .
$$

We have $E\left(\lambda_{0}\right)=0$ by assumption, and since the ground state represents always case $(\mathrm{A})$ in the terminology of [KS], in other words, zero is not an eigenvalue of $p^{2}+\lambda_{0} V$, we know that

$$
E(\beta)=-b(V)\left(\beta-\lambda_{0}\right)^{2}+\mathcal{O}\left(\left(\beta-\lambda_{0}\right)^{3}\right),
$$

holds for $\beta \geq \lambda_{0}$, close to $\lambda_{0}$, and for some positive constant $b(V)$ depending only on the potential $V$. Notice that the above asymptotic expansion coming 
from [KS, Theorem 2.3] was derived there for $V \in C_{0}^{\infty}\left(\mathbb{R}^{3}\right)$, however, an extension to the Rollnik class is straightforward. Recall now that

$$
\left|e_{\lambda}\right|=-(1-g(\alpha)) E\left(\lambda(1-g(\alpha))^{-1}\right) \text {. }
$$

Since $(1-g(\alpha)) \lambda_{0}<\lambda \leq \lambda_{0}$ holds by assumption and $g(\alpha)=\mathcal{O}(\alpha)$, we have

$$
\lambda(1-g(\alpha))^{-1}-\lambda_{0} \leq \lambda_{0} g(\alpha)(1-g(\alpha))^{-1}=\mathcal{O}(\alpha),
$$

and therefore

$$
\left|e_{\lambda}\right|=b(V)\left(\frac{\lambda}{1-g(\alpha)}-\lambda_{0}\right)^{2}+\mathcal{O}\left(\alpha^{3}\right)
$$

where the first term at the right-hand side is $\mathcal{O}\left(\alpha^{2}\right)$. Returning to (2.36) we conclude from the last claim that the second term in (2.36b) is of order of $\alpha^{3}$. This yields for all $\lambda$ in the considered range and for small $\alpha$ the asymptotic inequality

$$
E(\alpha, \lambda V) \leq E(\alpha, 0)-\left|e_{\lambda}\right|+\mathcal{O}\left(\alpha^{3}\right) .
$$

Since $b(V)>0$ the second term at the right-hand side is negative and dominates over the error for $\alpha$ sufficiently small. This demonstrates that the sought inequality (2.1) is valid under the assumptions we have made and thus it proves Theorem 1 .

\section{REFERENCES}

[AA] A. Arai, H. Kawano, Enhanced binding in a general class of quantum field models, Rev. Math. Phys. 15 (4), 387-423 (2003).

[BFS] V. Bach, J. Fröhlich, I.M. Sigal, Spectral analysis for systems of atoms and molecules coupled to the quantized radiation field, Commun. Math. Phys. 207 (2), 249-290 (1999).

[CH] I. Catto, Ch. Hainzl, Self-energy of one electron in non-relativistic QED, J. Funct. Anal. 207 (1), 68-110 (2004). Eprint arXiv/math-ph/0207036.

[CVV] T. Chen, V. Vougalter, S. Vugalter, The increase of binding energy and enhanced binding in nonrelativistic QED, J. Math. Phys. 44 (5), 1961-1970 (2003).

[GLL] M. Griesemer, E.H. Lieb, M. Loss, Ground states in non-relativistic quantum electrodynamics, Invent. Math. 145 (3), 557-595 (2001).

[H1] Ch. Hainzl, One non-relativistic particle coupled to a photon field, Ann. Henri Poincaré 4 (2), 217-237, (2003). Eprint arXiv/math-ph/0202001.

[H2] Ch. Hainzl, Enhanced binding through coupling to a photon field, In Mathematical results in quantum mechanics (Taxco, 2001), Contemp. Math. 307, 149-154, Amer. Math. Soc., Providence, RI (2002).

[HS] Ch. Hainzl, R. Seiringer, Mass renormalization and energy level shift in nonrelativistic QED, Adv. Theor. Math. Phys., 6 (5), 847-871 (2002).

[HVV] Ch. Hainzl, V. Vougalter, S.A. Vugalter, Enhanced binding in non-relativistic QED, Commun. Math. Phys. 233 (1), 13-26 (2003). Eprint arXiv/math$\mathrm{ph} / 0109015$.

[HHS] Ch. Hainzl, M. Hirokawa, H. Spohn, Binding energy for hydrogen-like atoms in the Nelson model without cutoffs. Eprint arXiv/math-ph/0312025.

[HiSp] F. Hiroshima, H. Spohn Enhanced binding through coupling to a quantum field, Ann. Henri Poincaré 2, 1159 (2001). 
[KS] M. Klaus, B. Simon, Coupling constant thresholds in nonrelativistic quantum mechanics. I. Short-range two-body case, Ann. Physics 130 (2), 251-281 (1980).

[RS] M. Reed, B. Simon, Methods of Modern Mathematical Physics. II. Fourier Analysis. Self-adjointness, IV. Analysis of Operators. Academic Press, New York-London, 1975, 1978.

CEREmade, CNRS UMR 7534, Université Paris-Dauphine, Place du MaréChal de Lattre de Tassigny, F-75775 Paris Cedex 16, France

E-mail address: catto@ceremade.dauphine.fr

Department of Theoretical Physics, NPI, Academy of Sciences, 25068 ŘĚ̌Prague, Czechia

E-mail address: exner@ujf.cas.cz

Ceremade, Université Paris-Dauphine, Place du Maréchal de Lattre de Tassigny, F-75775 Paris \& Laboratoire de Mathématiques Paris-Sud-Bat 425, F-91405 Orsay Cedex

E-mail address: hainzl@ceremade.dauphine.fr 